\title{
Top 10 Interventions in Paediatric Patient Safety
}

\author{
John Fitzsimons, MB, MSC, DipLQH, MRCPI, FRCPCH ${ }^{1,2, *}$ \\ David Vaughan, MB, FRCPI
}

\author{
Address \\ ${ }^{*}, 1$ Our Lady of Lourdes Hospital, Drogheda, Co. Louth, Ireland \\ Email: fitzsimonsQID@gmail.com \\ ${ }^{2}$ Health Services Executive, Dublin, Ireland \\ ${ }^{3}$ Hamad Medical Corporation, Doha, Qatar
}

Published online: 13 November 2015

(C) Springer International Publishing AG 2015

This article is part of the Topical Collection on Patient Safety

Keywords Paediatrics - Patient safety - Harm - Safety interventions - Quality improvement - Improvement science • Context

\section{Opinion statement}

Patient safety is at the core of modern healthcare. The concept of harm caused by the processes of healthcare is evolving, and injuries once seen as unavoidable are now seen as preventable and open to mitigation. There is growing evidence of safety interventions that can influence harm. In paediatrics, much of this evidence has been extrapolated from adult studies and the questions that must be asked are as follows: Do these interventions address the major harms seen in paediatrics? And will they work in paediatric populations? Equally important is being able to differentiate between the effect of the intervention and that of the context in which it is to be implemented; what are the foundational elements of patient safety that improve the chances of successful implementation and how do we promote the use improvement science as a means to change behaviour and embed best practice? Separating these features is complex and often leaves questions about what the key components of safety interventions are. An understanding of safety theory and improvement science is required if we are to make progress in the application of complex interventions across multiple care environments. We must find ways to share learning and collaborate with the purpose of accelerating the discovery, spread and application of top safety interventions in what is truly a global challenge.

\section{Introduction}

Patient safety has been marked as a key priority of healthcare in recent decades not only because of the recognition of the extent and severity of the problem but equally because of the demonstration that successful interventions can reduce, mitigate or prevent known harm [1]. The challenge for healthcare 
professionals and organizations is how to best match, prioritize and implement safety interventions that provide effective, evidence-based, relevant, achievable, measurable and best value protection from harm across the care spectrum. It is widely accepted that all healthcare staff have a professional and ethical obligation to ensure they provide the safest and highest quality care; what is less frequently acknowledged is that there is a similar ethical onus upon them to ensure that they implement safe practice on a foundation that maximizes the likelihood of success. Hence, it is important not just to identify those practices that will likely have a substantial impact but also to identify the other critical success factors and ensure they are also considered and put in place.

In 2013, the Agency for Healthcare Research and Quality (AHRQ) published a critical analysis of pa-

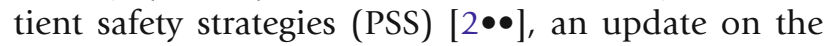
2001 report, "Making Health Care Safer: A Critical Analysis of Patient Safety Practices" [3]. The report defines a PSS as a type of process or structure whose application reduces the probability of adverse events resulting from exposure to the healthcare system across a range of diseases and procedures. The report, compiled by a panel of 20 safety experts, asked important questions to evaluate PSS including the burden of the problem, the nature and theory behind the intervention, potential benefits and harms, costs, ease of implementation and the impact of context. The authors conclude the report and an accompanying publication in the Annals of Internal Medicine with a suggested list of 22 PSS that are strongly encouraged or encouraged for implementation today [4]-Table 1 . While the report evaluates many paediatric studies and addresses problems relevant to paediatrics safety, it did not make specific paediatric recommendations.

The AHRQ report and other such evaluations of PSS are helpful in ensuring that interventions are evidence based. The AHRQ evaluation was also very mindful of the importance of context and other supporting elements; however, these may be overshadowed by the focus on the process and the pursuit of reliability of the PSS. Finally, when interventions are described as "top safety strategies" or "strongly encouraged", there is a suggestion that these practices should be prioritized above others.

So, how do we choose what our top paediatric patient safety interventions should be? We believe that there are three key drivers (Fig. 1) that successful safety interventions require and, like a bundle, all elements are required to accrue the benefit.

- First, we need to understand the need and the problems we seek to address.

- Second, there are foundational drivers, not specific to any safety intervention, but without them, it is likely that successful implementation, spread and sustainability will fail.

- Third, we must use knowledge of patient safety and quality improvement effectively and learn how to adapt the evidence into our particular context.

In this article, we will examine these drivers and their role in the generation of safety. We will also review the list of 22 PSS and their use from the point of view of relevance, paediatric evidence base and implementation challenges with a survey of members of the Pediatric International Patient Safety and Quality Community (PIPSQC).

\section{Measure and learn about harm and safe practice}

First do; know harm.

Identifying and understanding the nature of harm caused by healthcare provides the purpose for patient safety interventions [5]. The act of measuring and sharing data is in itself an important intervention if used to support safety behaviours. There are many methods used to measure harm and at their core are tradeoffs between the ease and the detail of data gathered.

\section{Local data on harm}

Trigger tools are a rapid and informing method to measure harm and have recently been adapted for use in paediatrics. Some have been adapted to specific paediatric settings and patient groups while others have focused on general (global) care. Trigger tools are designed and weighted towards the identification of common causes of harm, but they may also pick up other causes when broad definitions of harm are used [6]. What is emerging from the application of trigger tools is how frequently harm occurs in the paediatric inpatient hospital setting. Despite an increasing awareness of and focus on the need to improve safety, many experts remain concerned at what appears to be unacceptably slow progress in reducing preventable harm [7]. Recent publications have estimated that as many as one in four children 
Table 1. List of 22 patient safety practices from AHRQ report that can be strongly encouraged or encouraged because of supporting evidence

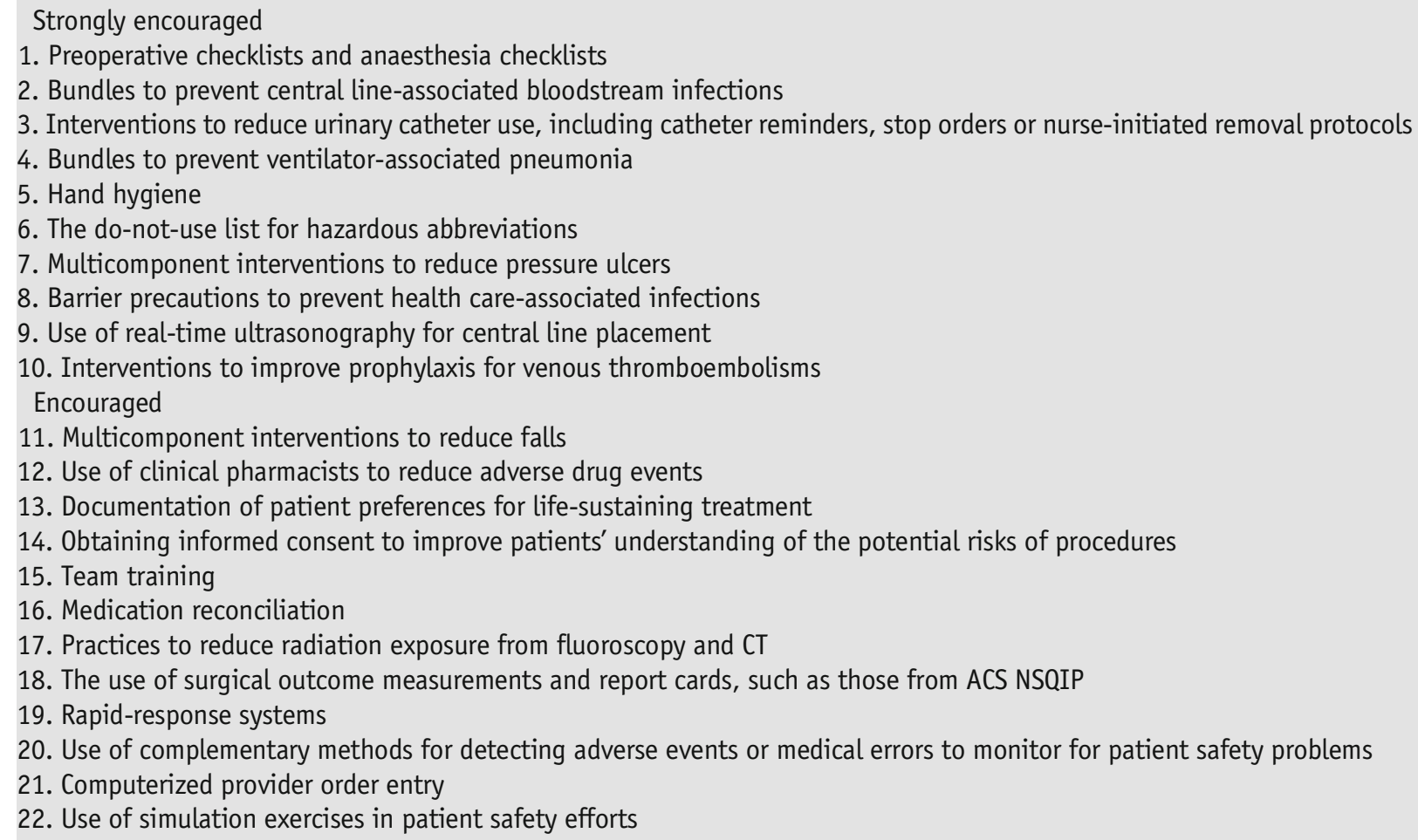

experience at least one episode of harm during hospitalization $[8 \bullet \bullet, 9]$.

Trigger tool studies have also provided insights into the specific causes of harm. Studies of medication adverse events have identified recurrent themes such as harm related to the administration of opiates, insulin and antibiotics [10, 11]. A trigger tool designed specifically for general paediatrics identified tissue injury related to intravenous cannulas as the most common cause of harm in hospitalized children, responsible for almost one in five harm events $[8 \bullet \bullet$.

\section{System-wide data on harm}

Measuring harm locally is important so that improvement efforts to reduce it can be guided and verified; however, these data can also be pooled to create a big picture view [6]. Other sources of big data include collaborative improvement networks that use multisite data to drive state-wide safety initiatives [12•].

A different kind of system-wide view comes from the UK's Confidential Enquiry into Maternal and
Child Health (CEMACH) [13]. The 2008 report of a sample of child deaths identified failures in a child's direct care in almost a quarter of all deaths, and potentially avoidable factors in up to half of inhospital deaths. Of particular concern in this report was the failure to recognize deteriorating children, with the report calling for the development of paediatric early warning systems.

Finally, national or state safety bodies that evaluate reports of harm provide an important opportunity to learn about infrequent but definite harms (such as hyponatraemia caused by the use of $0.18 \%$ saline solution) and provide safety recommendations [14].

Combining different types of data across sites and even nations provides a greater understanding of harm that allows for consensus on what the most prevalent and unwanted adverse events are in paediatric practice. This could also highlight events that are agreed to be preventable [15]. Table 2 includes a selection of features that could be considered as the goals of harm-free paediatrics. 


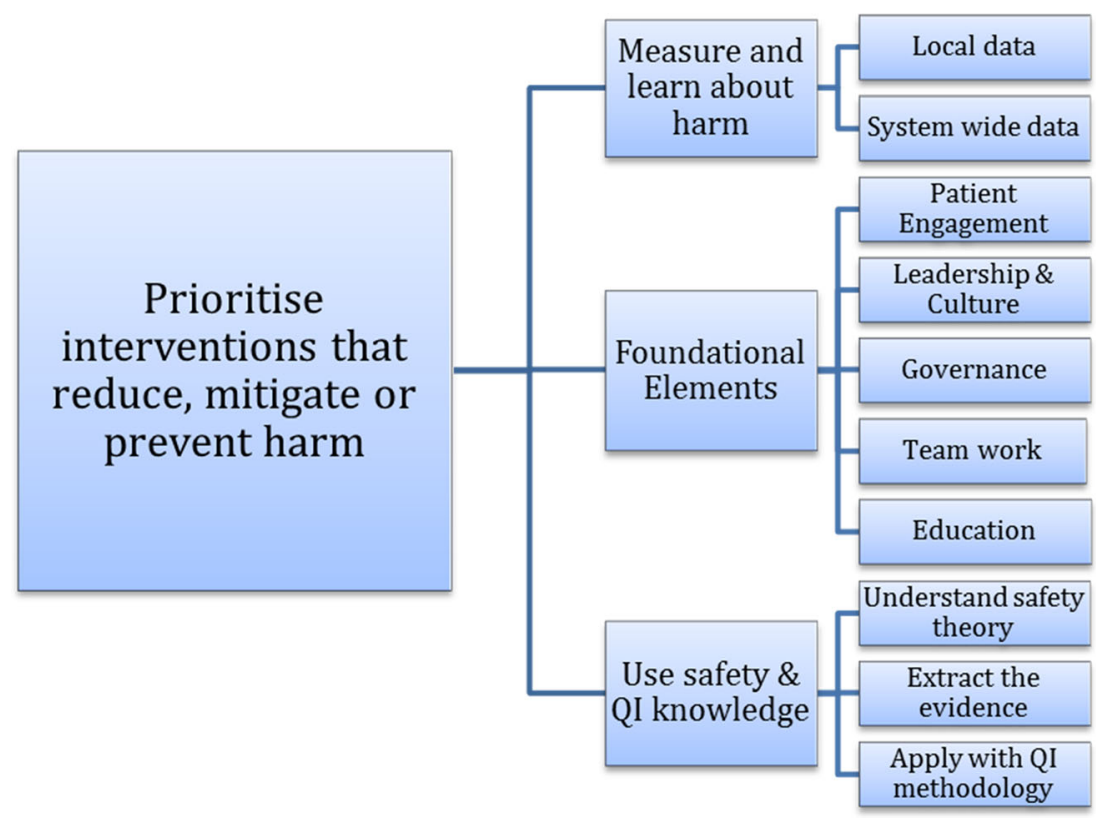

Fig. 1. Drivers to prioritize inventions that reduce, mitigate or prevent harm.

\section{Foundational elements of safety}

The foundational elements of patient safety are, we believe, those features that are responsible for what is collectively described as context when evaluating PSS. Context may been defined as "everything that is not the intervention" [16]. These are the factors that are responsible for the engagement, belief, effort and support required for successful implementation and sustainability. Focusing on internal factors only, we chose five important elements although it may be argued that there are many others [17].

\section{Table 2. The goals of harm-free paediatrics}

\section{0 suggestions for harm-free paediatrics}

1. No or minimal pain and distress

2. No tissue injury-extravasation, pressure ulcer or other tissue injury

3. No hospital-acquired infections

4. No medication or fluid injuries

5. Early recognition and management of procedural or surgical complications

6. Early recognition and management of sepsis or other life-threatening illnesses

7. Early recognition and management of in-hospital deterioration

8. Early recognition and management of safeguarding concerns

9. No unnecessary admissions, investigations, procedures or treatments

10. No psychological harm—provide a positive experience 
- Patient engagement

- Leadership and culture

- Governance

- Teamwork

- Education

Bohmer emphasizes the interdependency of these elements in creating purpose for change when he describes what it is medical leaders need to communicate, "It is the value of patient benefit, patient autonomy and professional excellence that must serve as the compass of healthcare reform" [18]. The interaction between genuine patient engagement and strong clinical leadership is a key factor in the generation of a culture of patient safety.

Less commonly stressed is the importance of good governance, both at the macrosystem and microsystem levels. Good governance ensures that management systems, structures and processes are in place to provide accountability and assure the delivery of safe and high-quality care [19]. It also needs to challenge the blind pursuit of, sometimes counter-productive, externally imposed targets or assurance mechanisms from regulators and payers. [20].

Teamwork and education may be seen as traditional factors; however, the complexity and rate of change in modern healthcare mean they must evolve rapidly. Advances in human factors psychology, simulation and information technology provide opportunities to match training and teaching experiences to the needs of today.

We believe that resourcing these five foundational elements addresses many of the enablers and barriers of context in the successful implementation of patient safety practices. In fact, it is likely that these must be in place and functional in advance of any successful and sustainable safety intervention.

There remains a final foundational element that we have chosen to examine as a separate driver, one that helps to understand context but that may also compensate for and support the creation of positive context. Knowledge of patient safety and quality improvement can be used to learn much about what some authors consider a more useful definition of context, "the complex nature of local care settings - their processes, habits, and traditions" [21]

\section{Use safety and quality improvement knowledge and capability}

At the heart of choosing paediatric patient safety interventions is the question of what constitutes evidence. Is it simply evidence of efficacy? That the intervention can work. Or is evidence of reliable application and errorfree implementation also required? That the proven intervention can be applied appropriately, in real world settings, and implemented flawlessly. Disentangling the practice from the system is a complex task [22].

While some of the effects of context can be addressed when assessing PSS, many remain and must be acknowledged. This is especially true when evaluating interventions in paediatrics as appraisals of safety interventions may include, but are rarely exclusive to, paediatric studies. Therefore, judgement is required about whether the intervention addresses a significant problem in paediatric care. Does it need a paediatric evidence base because of differences in physiology or pathology? And are the contextual factors different if applied in a 
paediatric setting? Generating evidence that can address all of these uncertainties is a tall order and only ever likely for a very select number of problems. It is possible, however, to compensate for many of these deficits by using knowledge and expertise of patient safety and quality improvement to assess and apply existing evidence. Investing in these skills is also likely to improve the quality of new evidence as research and quality improvement knowledge combines [23].

The understanding of the theory behind harm and safety provides a significant benefit to those evaluating interventions. Patient safety theory (the concepts and models explaining the cause of harm and the source of safety) is evolving with inputs from many disciplines from within and outside of medicine. This evolution has seen a change in views of how harm occurs, in particular from harm being attributed to an individual's actions to harm being seen as a system attribute [24]. This in turn has influenced new safety interventions and supporting practices (see Table 3 ).

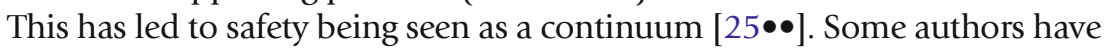

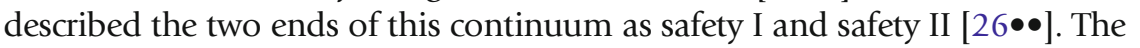
safety I end, familiar to most practitioners, portrays harm as a clear cause and effect relationship with interventions targeted at easily identified defects. The safety II end is focused on recognizing the adaptability that practitioners apply to allow everyday work to happen successfully. Many of the ideas of safety II are shared with resilience engineering, and while they have been used to describe safe systems, their practical application is yet to be established [27]. In between, however, are a myriad of concepts and models that move from simple to complex interventions, including risk management, highreliability organizations (HROs) and resilient teams. This does not mean an abandonment of safe practices that are proven to be effective; however, it does signal the need for more sophisticated means of evaluation for complex or whole system changes.

The importance of understanding context has been highlighted by a number of high-profile publications suggesting that proven safety interventions are less effective when implemented in other settings $[28,29]$. This often leads to questions about the efficacy of the intervention in the first place and is usually matched with claims of poor compliance, leadership or organizational buy-in at new sites [29, 30]. An understanding of quality improvement methodology provides an insight into the implementation

Table 3. The safety continuum. Models and concepts

\begin{tabular}{|c|c|c|c|c|c|}
\hline \multirow[b]{2}{*}{$\begin{array}{l}\text { Example safety } \\
\text { interventions }\end{array}$} & \multicolumn{5}{|c|}{ Safety continuum } \\
\hline & & $\begin{array}{l}\text { Remove } \mathrm{KCl} \text { from } \\
\text { fluids drawers }\end{array}$ & CVC bundles & $\begin{array}{l}\text { Paediatric early } \\
\text { warning system }\end{array}$ & \\
\hline Design principles & $\stackrel{n}{\stackrel{\infty}{\infty}}$ & Design out error & $\begin{array}{l}\text { Design for } \\
\text { reliability }\end{array}$ & Design in resilience & $\underset{\mathscr{D}}{\stackrel{n}{D}}$ \\
\hline Human factors & $\stackrel{\mathbb{P}}{<}$ & People are a safety risk & Train for safety & People generate safety & 䒿 \\
\hline Risk principles & $\mapsto$ & Avoid risk & Manage risk & Embrace risk & 曰 \\
\hline $\begin{array}{l}\text { Leadership and } \\
\text { governance }\end{array}$ & & Authority and accountability & & Engagement and learning & \\
\hline
\end{tabular}




\section{Ask the experts}

strategies and the foundational elements that were mobilized to support the intervention.

Finally, quality improvement knowledge provides for the development and testing of new implementation strategies that might support an effective intervention. Understanding and applying design methods to reduce the burden of trying new interventions is likely to improve the chances of success [31].

As part of this paper, we wanted to assess the usefulness of the AHRQ list of 22 PSS for use in a paediatric population. We had initially evaluated this list ourselves but believed a broader view was required so we consulted an "expert" panel. In June 2015, we conducted a non-targeted survey of members of the Pediatric International Patient Safety and Quality Community (PIPSQC) about their interpretation of the PSS included in the AHRQ report and what they regarded as the top patient safety interventions.

We asked participants three questions regarding the AHRQ list of PSS. Five options were provided for each question.

1. What is the relevance of the intervention in paediatrics? (Not relevant to relevant for most, 1-5)

2. How strong is the paediatric evidence base for the use of the intervention? (No evidence to good evidence, 1-5)

3. What is the degree of difficulty in implementing this intervention? (Easy to difficult, 1-5)

We also asked respondents to rank their top three paediatric patient safety interventions, which could include interventions from the AHRQ list of 22. We received 60 survey responses with respondents from six countries (USA, Canada, UK, Ireland, Australia and New Zealand). The majority of respondents were nurses or doctors (38 and $40 \%$ respectively) with pharmacists, allied health and administrative staff making up the remainder.

The survey results summarized in Table 4 provide an interesting insight into PSS in paediatrics and support many of our observations.

- More than $50 \%$ of respondents ranked 20 of the 22 PSS as relevant to many or most paediatric cases

- More than $75 \%$ of respondents ranked half of PSS as relevant to many or most paediatric cases

Medication safety, not surprisingly, topped the list of freely chosen safety interventions reflecting its importance in paediatric patient safety. This covers a broad range of harm causes and a correspondingly broad number of interventions required to generate safety that goes beyond those evaluated on the AHRQ list. Access to clinical pharmacists ranked highly as a top intervention.

It is interesting that hand hygiene, possibly healthcare's original patient safety strategy, features so prominently. An intervention that is universally accepted, cheap and effective but seen as both easy and difficult to implement, highlights the challenges of all safety interventions. 


\section{Table 4. PIPSQC survey results}

Relevant in most cases

1. Hand hygiene

2. Team training

3. Clinical pharmacists

4. Infection barriers

5. CVL bundles

6. Pre-op checklist

7. Consent

8. Do-not-use abbreviations

9. Rapid-response teams

10. Medication reconciliation

Least relevant

Falls prevention

Venous thomboembolism prophylaxis

Pressure ulcers

Easy implementation

Do-not-use abbreviations

Hand hygiene

Consent

Top 3 (including PSS)

1. Medication safety (including availability of clinical pharmacists)

2. Team training and communication

3. Hand hygiene
Enough or good evidence in paediatrics

1. Hand hygiene

2. Central venous line (CVL) bundles

3. Infection barriers

4. Clinical pharmacists

5. Team training

6. Pre-op checklists

7. Simulation

8. Ventilator-associated pneumonia bundles

9. Rapid-response teams

10. Complementary measures of patient safety

Little or no evidence

Falls prevention

Venous thomboembolism prophylaxis

Do-not-use abbreviations

Difficult implementation

Computer physician order entry

Team training

Alternative measurement

\section{Conclusion}

So what are the top 10 interventions in paediatric patient safety? Are they those that address the most common or most severe causes of injury that could lead to harm-free paediatrics? Are they the 10 drivers that lead to successful prioritization and implementation of safe practice? Or are they the 10 patient safety strategies adapted from evidence-based evaluation and selected by our paediatric safety experts?

How you answer this question is likely to depend on your context. There is no single right answer. In contrast to the adult interventions in the paper by Shekelle et al., we do not believe there exists a prescriptive list of interventions to be addressed. Even if such a list could be compiled, the danger is that payers, regulators and others will focus on these simple interventions to the exclusion of others and thus perpetuate the systems failings that contribute to the majority of harm and error. Many interventions are only applicable to hospital settings and then even in specialized sections of the hospital such as PICU. Others, such as hand hygiene, are relevant in all settings and are simply foundations of good practice. 
It is important to acknowledge that there has been relatively little research into the burden of medical harm occurring in the community or at home; however, studies that have examined this have found comparable or higher levels than those found in studies of hospitalized patients [32]. This is an important area of study as more care moves to the community.

We believe paediatric safety advocates must be equipped to help them address this question adequately. It may be that they can pick from a list of tried and tested interventions that have been proven in similar and specific environments and adapt with minimal changes. Some interventions should be named as standards, globally accepted, expected and reliably implemented everywhere because not doing so would be medically negligent or ethically unacceptable. Practices such as the use of central venous line bundles have a sufficient evidence base to be seen in this light. Support from foundational elements and quality improvement methods are still required and need to be seen as part of the intervention "package" when being resourced.

Addressing the majority of hazards and known harms will require more tailored solutions; based on an understanding of the nature of the problem, the theory behind interventions and the ability to adapt to local context. As opposed to other areas of science or medicine where evidence may be seen as a recipe to generate a specific outcome, in safety and improvement, evidence of effect remains only an important ingredient.

A fundamental rule of improvement is that healthcare is a complex system; focusing on simple interventions and expecting that these will result in a transformation is not consistent with a true systems perspective. It is, however, essential that the international healthcare community continues to research and evaluate safety interventions to show that specific harms can be diminished but also that we develop better methods to understand why success occurs.

The importance of the foundational elements in all improvement activity, and the fact that they are responsible for many of the positive effects of context in the successful implementation of interventions, needs to be better recognized and studied. These foundational elements also contribute to why so much activity goes right without the need for specific safety interventions. Understanding these roles and identifying which other factors are responsible for things going right is likely to provide significant insights into the future direction of patient safety.

We must continue to learn how to extract and use the good ideas from existing studies beyond the reporting of effect alone while encouraging new studies to make the theories of success more explicit [33••]. This will require an investment in capability and capacity for improvement but one that will be returned many times with better quality and safety outcomes [34].

Finally, we must continue to measure and share our learning. Paediatric patient safety communities, networks and collaboratives are leading the way in how to work together to improve paediatric patient safety. The Pediatric International Patient Safety and Quality Community (PIPSQC, www.pipsqc.org) uses social media to connect like-minded clinicians around the globe, and groups such as the Children's Hospitals' Solutions for Patient Safety (www.solutionsforpatientsafety. org) Network in the USA or the Making It Safer Together (www.mistuk.org) paediatric patient safety collaborative between children's hospitals in the UK and Ireland are transforming and accelerating how we learn about harm and safety. Together, we can make harm-free paediatrics a real goal. 
With special thanks to Dr. Genevieve Paisley and all the PIPSQC members who replied to the paediatric patient safety survey.

\section{Compliance with Ethical Standards}

\section{Conflict of Interest}

John Fitzsimons declares that he has no conflict of interest.

David Vaughan declares that he has no conflict of interest.

Human and Animal Rights and Informed Consent

This article does not contain any studies with human or animal subjects performed by any of the authors.

\section{References and Recommend Reading}

Papers of particular interest; published recently; have been

highlighted as:

- Of importance

$\bullet \quad$ Of major importance

1. Steering Committee on Quality Improvement and Management and Committee on Hospital Care. Policy statement-principles of pediatric patient safety: reducing harm due to medical care. 2011; 127(6):1199210.

2.• Shekelle PG, Wachter RM, Pronovost PJ, et al. Making health care safer II: an updated critical analysis of the evidence for patient safety practices. Comparative Effectiveness Review No. 211. AHRQ Publication No. 13E001-EF. Rockville, MD: Agency for Healthcare Research and Quality. March 2013.

Extensive evaluation of evidence base for patient safety practices.

3. Shojania KG, Duncan BW, McDonald KM, et al. Making health care safer: a critical analysis of patient safety practices. Evidence report/technology assessment No. 43, AHRQ publication No. 01-E058. Rockville: Agency for Healthcare Research and Quality; 2001.

4. Shekelle PG, Pronovost PJ, Wachter RM, et al. The top patient safety strategies that can be encouraged for adoption now. Ann Intern Med. 2013;158(5 Pt 2):365-8.

5. Walsh KE, Bundy DG, Landrigan CP. Preventing health care-associated harm in children. JAMA. 2014;311(17):1731-2.

6. Chapman SM, Fitzsimons J, Davey N, Lachman P. Prevalence and severity of patient harm in a sample of UK-hospitalised children detected by the paediatric trigger tool. BMJ Open. 2014;4(7), e005066.

7. Landrigan CP, Parry GJ, Bones CB, et al. Temporal trends in rates of patient harm resulting from medical care. N Engl J Med. 2010;363(22):2124-34.

8.• Stockwell et al. A trigger tool to detect harm in pediatric inpatient settings.; originally published online May 18, 2015; DOI: 10.1542/peds.2014-21520.

Description of tool to measure harm in inpatient settings providing insights into the causes of harm in pediatrics.

9. Kirkendall et al. Measuring adverse events and levels of harm in pediatric inpatients with the Global Trigger Tool. 2012; 130(5):e1206-14.

10. Muething SE, Conway PH, Kloppenborg E, Lesko A, Schoettker PJ, Seid M, et al. Identifying causes of adverse events detected by an automated trigger tool through in-depth analysis. Qual Saf Health Care. 2010;19(5):435-9.

11. Takata GS, Taketomo CK, Waite S. Characteristics of medication errors and adverse drug events in hospitals participating in the California Pediatric Patient Safety Initiative. Am J Health Syst Pharm. 2008;65(21):203644.

12. $\quad$ Billett AL, Colletti RB, Mandel KE. Exemplar pediatric collaborative improvement networks: achieving results. Pediatrics. 2013;131 Suppl 4:S196-203.

Report on the benefits of a collaborative improvement network.

13. Pearson, GA (Ed) Why children die: a pilot study 2006; England (South West, North East and West Midlands), Wales and Northern Ireland. London: CEMACH. 2008.

14. Patient safety alert 22: reducing the risk of hyponatraemia when administering intravenous infusions to children. National Patient Safety Agency, 2007. http://www.nrls.npsa.nhs.uk/resources/?entryid45= 59809 (Accessed 25th July 2015). 
15. Brilli RJ, McClead Jr RE, Davis T, Stoverock L, Rayburn A, Berry JC. The preventable harm index: an effective motivator to facilitate the drive to zero. J Pediatr. 2010;157(4):681-3.

16. Ovretveit JC, Shekelle PG, Dy SM, et al. How does context affect interventions to improve patient safety? An assessment of evidence from studies of five patient safety practices and proposals for research. BMJ Qual Saf. 2011;20(7):604-10.

17. Taylor SL, Dy S, Foy R, et al. What context features might be important determinants of the effectiveness of patient safety practice interventions? BMJ Qual Saf. 2011;20(7):611-7.

18. Bohmer R. The instrumental value of medical leadership: engaging doctors in improving services. London: The King's Fund; 2012.

19. Jha A, Epstein A. Hospital governance and the quality of care. Health Aff. 2010;29(1):182-7.

20. Meyer GS, Nelson EC, Pryor DB, James B, Swensen SJ, Kaplan GS, et al. More quality measures versus measuring what matters: a call for balance and parsimony. BMJ Qual Saf. 2012, 14.

21. Stevens DP, Shojania KG. Tell me about the context, and more. BMJ Qual Saf. 2011;20(7):557-9.

22. Leape LL, Berwick DM, Bates DW. What practices will most improve safety? Evidence-based medicine meets patient safety. JAMA. 2002;288(4):501-7.

23. Portela MC, Pronovost PJ, Woodcock T, et al. How to study improvement interventions: a brief overview of possible study types. BMJ Qual Saf. 2015;24(5):32536.

24. Emanuel L, Berwick D, Conway J, et al. What exactly is patient safety? In: Henriksen K, Battles JB, Keyes MA, et al., editors. Advances in patient safety: new directions and alternative approaches (vol. 1: assessment). Rockville: Agency for Healthcare Research and Quality; 2008.

25.• Vincent C, Amalberti R. Swiss Re Centre for global dialogue. Sept 2014. http://cgd.swissre.com/risk_
dialogue_magazine/Safety_management/A_ continuum_of_safety_models.html (accessed 25th July 2015).

New and important insights into patient safety theory and practice.

26.• Hollnagel E, Wears RL, Braithwaite J. From safety-I to safety-II: A white paper. The Resilient Health Care Net: Published simultaneously by the University of Southern Denmark, University of Florida, USA, and Macquarie University, Australia.

New and important insights into patient safety theory and practice.

27. Fairbanks RJ, Wears RL, Woods DD, et al. Resilience and resilience engineering in health care. Jt Comm J Qual Patient Saf. 2014;40(8):376-83.

28. Urbach DR, Govindarajan A, Saskin R, Wilton AS, Baxter NN. Introduction of surgical safety checklists in Ontario, Canada. N Engl J Med. 2014;370:1029-38.

29. Dixon-Woods M, Leslie M, Tarrant C, Bion J. Explaining Matching Michigan: an ethnographic study of a patient safety program. Implementation Science: IS. 2013;8:70.

30. Leape LL. The checklist conundrum. N Engl J Med. 2014;370:1063-4.

31. Hayes CW, Batalden PB, Goldmann D. A "work smarter, not harder" approach to improving healthcare quality. BMJ Qual Saf. 2015;24(2):100-2.

32. Walsh KE, Roblin DW, Weingart SN et al. Medication errors in the home: a multisite study of children with cancer. 2013; 131(5): e1405-14.

33.• Davidoff F, Dixon-Woods M, Leviton L, Michie S. Demystifying theory and its use in improvement. BMJ Qual Saf. 2015;24(3):228-38.

Good review on use of theory to support improvement.

34. Lannon CM, Levy FH, Moyer VA. The need to build capability and capacity in quality improvement and patient safety. 2015; 135(6):e1371-3. 